\title{
Towards Better Semantics for Services in eHealth Standards: A Reference Ontology Approach
}

\author{
Zoran Milosevic ${ }^{1}$, João Paulo A. Almeida ${ }^{2}$, Julio C. Nardi ${ }^{2,3}$ \\ ${ }^{1}$ Deontik Pty Ltd, Brisbane, Australia \\ ${ }^{2}$ Ontology \& Conceptual Modeling Research Group (NEMO), Federal University of Espírito Santo, Vitória, Brazil \\ ${ }^{3}$ Informatics Department, Federal Institute of Espírito Santo, Colatina, Brazil \\ zoran@deontik.com,jpalmeida@inf.ufes.br,julionardi@ifes.edu.br
}

\begin{abstract}
Responding to a wide range of interoperability requirements for eHealth, the HL7 standardization organization has recently published an initial version of the SOA Healthcare Ontology (SHO). The purpose of this effort is to serve as a shared reference conceptual model, which can guide the definition of service-related aspects of future eHealth standards and solutions. In this paper, we analyse the SOA Healthcare Ontology using a well-founded ontological approach referred to as UFO-S. UFO-S is a reference ontology that characterizes the notion of service by applying the concepts of commitments and claims and harmonizing several views of services from a broad perspective. Our objective is to provide an ontological foundation to this new HL7 standardization effort. Given that UFO-S is formalized and defined according to a sound foundational ontology, we believe this paper can serve as a basis for further improvement in the formalization and revision of SHO and its conceptual representation.
\end{abstract}

Keywords: Service-oriented architecture; Service Ontology; Enterprise architecture; eHealth standards.

\section{INTRODUCTION}

Last decade has seen a significant broadening of eHealth landscape from the traditional health informatics concerns to a wider range of interoperability requirements [18][19][27]. Health informatics is mostly focused on modelling information to describe clinical concepts related to the health status or conditions of patients. This information is captured for example by clinicians through clinical observations recorded in clinical documents, which can be stored in electronic health records, or as part of payload of messages exchanged between laboratory systems and general practitioners (GP) desktop systems. Last two decades have seen significant progress in standardizing clinical concepts needed for such information modelling, as published through many specifications available at the Health Level Seven International (HL7), ANSI-accredited standards developing organization [20]. This emphasis on information semantics is an important contributor to addressing information interoperability requirements.

However, the increasing use of IT systems to support information exchange and care coordination across different healthcare providers, often across organizational and jurisdictional boundaries, requires new eHealth approaches for modelling and implementing behavioural semantics [19]. This involves at least two challenges. Firstly, there is a requirement for establishing a common agreement on enterprise concepts such as process, policy, organizational boundary and service. Second, there is a need to clearly articulate how clinical information concepts and the supporting technical infrastructure are used in such an enterprise context.

Consider for example an eHealth support for exchanging referral documents from one clinician to another (what is called e-Referral). This requires the expression of data elements which constitute a traditional referral document, such as the names of referring and referred-to clinicians, the purpose of the referral and the name of patient, her health status description and requirement for specific health intervention. This constitutes information semantics aspects of eReferral. While this electronic version of referral can be exchanged via simple electronic facilities such as faxes and emails, this is not a scalable solution, in particular when one needs to allow a referring clinician to look up electronically for most suitable and currently available referred-to clinicians, using for example an electronic directory of healthcare service providers [21]. This can be further complicated by organizational policies, e.g. only certain roles in the receiving organization are permitted to open the referral document, and this may vary from one type of referral to another, depending on its content sensitivity. Another requirement may be that the referring doctor would like to be notified about the progress of care or status of the intervention and may expect a report from the referred-to provider (e.g. a cardiologist) about the outcome of their treatment. Further, a vendor providing electronic directory of healthcare services is governed by various types of contractual conditions placing commitments on them while also allowing claims to those who are using its service, both referring and referred-to clinicians.

The above simplified example illustrates the behavioural aspects of an eHealth system (as part of a broader health system) including various constraints on behaviour, most notably those specified in enterprise policies. It also highlights its link to the information aspects of eHealth systems. Note that we have omitted here technical infrastructure details such as an access control mechanism used within referred-to organization, an exchange protocol or a hosting component of the directory service, but these need to be considered in developing a complete eReferral solution architecture.

The main focus of this paper is on the behavioural aspects of eHealth systems with the purpose of identifying key concepts that characterize an eHealth service as part of a 
broader eHealth system which itself is part of a broader healthcare. Our objective is to analyse current approach to specify eHealth service semantics, as proposed in a recent HL7 publication [21], through applying a general, commitment-based service ontology, Unified Foundational Ontology for Services (UFO-S) [30] as a reference ontology. We chose UFO-S because of its account for services through commitments associated with service participants, such as healthcare providers, patients, eHealth providers etc. We aim at testing the expressiveness of the HL7 SOA Healthcare Ontology and thus provide a better ontological foundation to this new HL7 standardization effort. The ultimate goal is to develop a well-founded semantics and agreement on the concept of service particularly suited for eHealth applications. Another aim is to consider eHealth service requirements and potentially identify areas within UFO-S that need further development.

The remainder of paper is structured as follows. Section II introduces key service concepts as defined in the HL7 SOA healthcare ontology. Section III presents the core reference ontology UFO-S. Section IV provides an analysis of the alignment between the UFO-S and HL7 SOA Healthcare Ontology concepts. Section V discusses the various roles that ontologies can play in standardization efforts, positioning the work reported in this paper with other efforts in the literature. Section VI concludes the paper and indicates potential areas of further research.

\section{HealthCARE SERVICES ONTOLOGY}

HL7 SOA Healthcare Ontology (referred to SHO in the reminder of this paper) standardization efforts aim at establishing a common semantics for the concept of eHealth service [21]. This is needed as part of increasing interoperability both between people involved in designing eHealth solutions, between eHealth systems and between people and systems. The status of the SHO specification is 'for comment only ballot', which allows for submitting feedback to improve and consolidate this specification before next stages in standardization. This paper can be provided as input in this standardization effort.

SHO was developed to leverage the HL7 Service Interoperability Framework (SAIF) [19], which in turn was based on key architectural principles from the ISO/IEC RMODP standard [18] and on OMG's MDA framework [34]. $\mathrm{SHO}$ has two main purposes. Once the eHealth service semantics is agreed, the SHO can be used to facilitate building eHealth solution architectures requiring support for implementing eHealth services, including various kinds of commitments and claims that they impose on the parties involved. It can also be used as a basis for developing various kind of taxonomies, which can be employed within enterprises when establishing their own service catalogues, needed for enterprise architecture activities, such as the establishment of service portfolio needed to support better reusability and consistency of eHealth services across the enterprise.
SHO makes use of the concept of service introduced in the SAIF [19] and RM-ODP [18]. The SHO specification is centred on the adoption of a generic concept of service so that it can be further refined to reflect the enterprise and computational views on service. Accordingly, service is defined as: "behaviour triggered by an interaction, that adds value for the service users by creating, modifying or consuming information; the changes become visible in the service provider's environment." [24]. Note that the provider's environment includes the service user and the last sentence suggests that provision of a service involves some kind of commitment by the provider to stand by its actions; they are seen by at least some things outside it, so the action is subject to audit. SHO adopted this interpretation of service to meet the eHealth requirements, namely the need to describe the IT infrastructure and IT services in support of coordinated healthcare delivery. The definition is generic enough however and it can be also used to describe healthcare services if required, e.g. a clinical service provided by a clinician to a patient.

It is important to highlight the fact that the definition above treats the concept of service as an instance of a realworld thing that involves interactions between service users and service providers. It implies that there is an implicit or explicit agreement between them (i.e. a service contract). This should be distinguished from a service description which is a concept used to express what service provider offers to the environment to facilitate service users' understanding of what value service will deliver to them. Note that, in RM-ODP, service description can be expressed using the concept of template, defined 'the specification of the common features of a collection of $\langle X>s$ in sufficient detail that an $\langle\mathrm{X}>$ can be instantiated using it', e.g. a Service Level Agreement form that can be completed with specific details of parties to the contract and contract conditions.

The diagram in Figure 1 depicts key modelling concepts for business service as proposed in the SHO [21]. It uses the UML notation to convey the semantics of SHO concepts and their relationships, i.e., its conceptual model.

The business emphasis highlights enterprise viewpoint concerns on service in line with the SAIF and RM-ODP enterprise viewpoint scope, while refining the generic concept of service, service contract and service description as introduced above.

A business service is an elaboration of the concept of service described above, including the following additional concepts and refinements:

- Service provider and service consumer roles - which are involved in service delivery; each of these roles is to be filled by a party, represented an organization or a person for example

- Party is a special kind of enterprise object that models a natural person or any other entity considered to have some of the rights, powers and duties of a natural person [25]. 


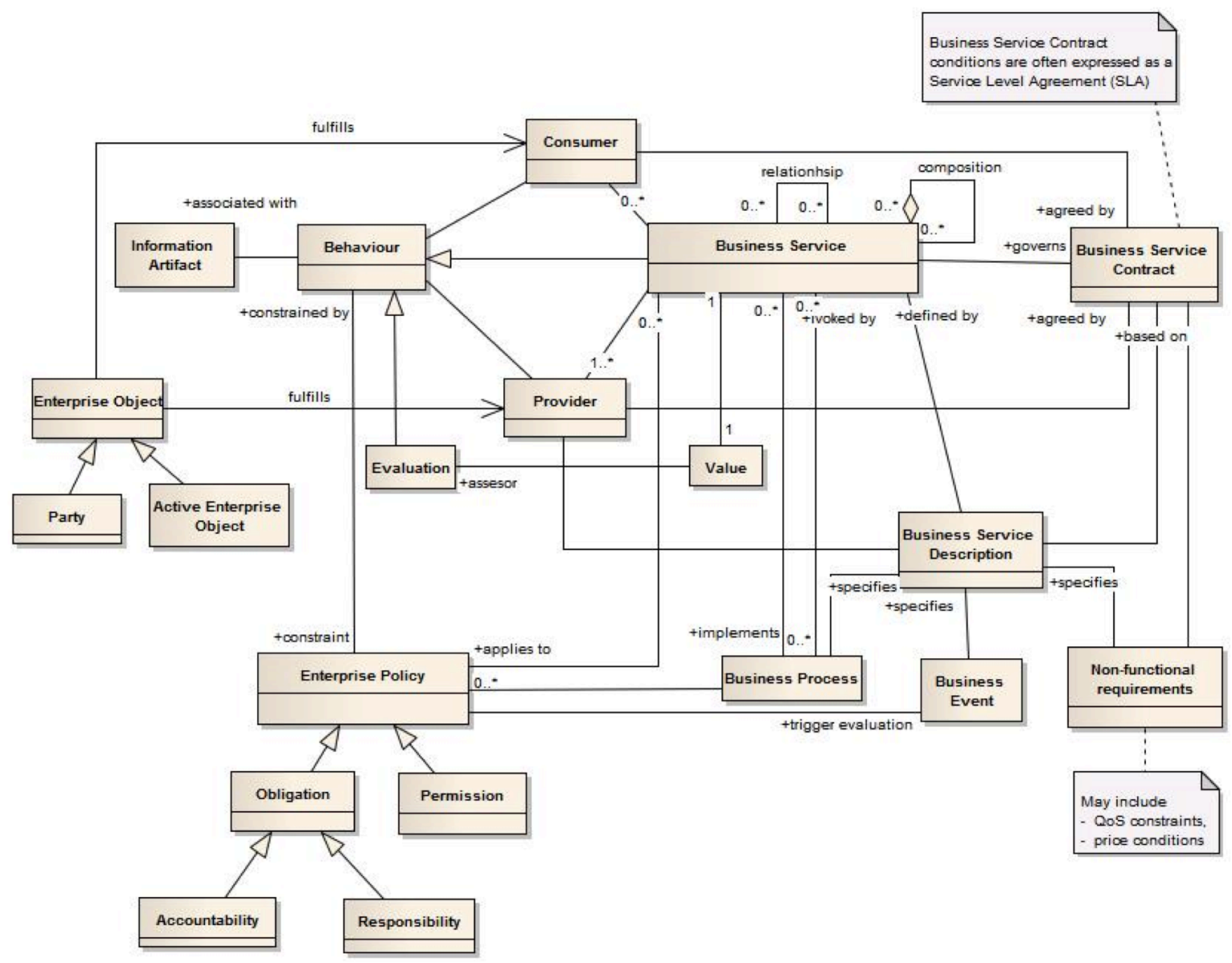

Figure 1: Modelling concepts for Business Services in HL7 SOA Healthcare Ontology

- A business service can be invoked from a business process but can also be realised through business process, such as in case of eReferral service. A business process is defined as 'A collection of steps taking place in a prescribed manner and leading to an objective' [24]. Note that for the purpose of eHealth service modelling, the specific details of particular process language are omitted, but could be added if use cases require so.

- A business service needs to be compliant with enterprise policies that apply to the business service. Typically, deontic policies such as obligations, permissions, prohibitions and authorisations would state constraints on the use or management of business services [25]. These can in turn provide a foundation for describing more complex policies such as responsibility, accountability, consent, privacy and duty of care, each of which can be expressed as a combination of obligations and permissions. Note that privacy and consent policies that might apply to eHealth systems are further discussed in the Privacy, Access and Security Services project [22].

- An information artefact represents, e.g., documents exchanged or consumable resources that are essential in delivering services; note that some of these may not require IT support but nonetheless would need to be identified in an enterprise viewpoint of a service model.
- Evaluation is a concept introduced to be able to measure value that a business service delivers to service users, and is defined as 'an action that assess the value of something' [25].

A business service description specifies expected business service behaviour, which captures expected collaboration between consumer and provider roles, which is typically expressed through a business process description. The description can also specify metrics or other data needed for service monitoring and assessment, including significant business events (e.g. payment) and non-functional service properties (e.g. service level agreements). The business service description can be advertised by service providers through service offers in a publicly accessible directory. Consumers can inspect these descriptions and accept the conditions or negotiate further tailoring for their requirements. The availability of service taxonomy is expected to facilitate lookup and search of business service descriptions.

A Business service contract formalizes and governs business service (delivery), once an agreement is reached on the business service description between a service provider and consumer. In many industries the term Service Level Agreement is used to describe the operational and service quality constraints associated with a service contract. Note 


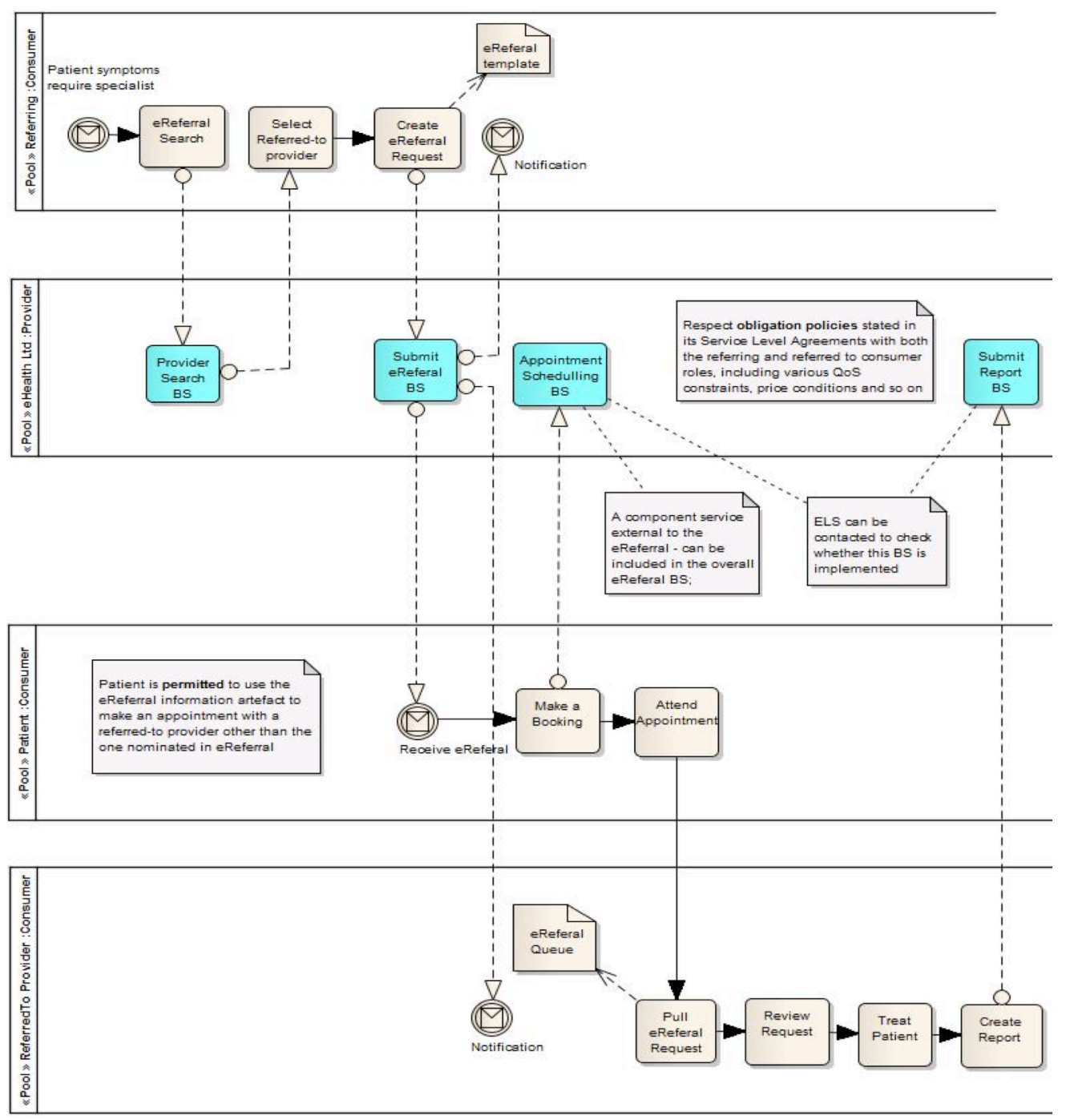

Figure 2: eReferral process, its business services and policies (from [21])

that a business service contract will need to include specific values related to the non-functional properties such as Quality of Service (QoS) requirements, price and so on.

Figure 2 illustrates several eHealth business services involved in eReferral process [21] used for handing over patent's health care from a referring to a referred-to healthcare providers, as mentioned in section I. These business services (depicted as tasks in blue colour) are offered by technology provider (provider role) and used by referring and referred-to providers and patient (consumer roles). Each business service provides a different value to a different type of consumer. For example, it provides value to patients in supporting better care as the electronic referral allow faster, more reliable and consistent information exchanges between health providers. The diagram also depicts examples of enterprise policies that provide constraints on the actions of object fulfilling roles in the business process and they are included within comments as part of the appropriate BPMN pools.

\section{UFO-S: CORE REFERENCE ONTOLOGY FOR SERVICES}

UFO-S was proposed in [30] as a core reference ontology for services based on the notion of social commitments. As a reference ontology [14], UFO-S is intended to assist humans in developing a shared understanding. We present UFO-S here briefly in order to use it as a semantic foundation to analyse SHO concepts later in section IV. For further information on UFO-S, see [30].

UFO-S is grounded in a foundational ontology (the Unified Foundational Ontology - UFO [15][16][17]), from which it reuses foundational notions of objects, types, object properties, object relations, events/processes, and further social concepts that specialize the more general notions and account for social reality. The social layer of UFO includes important notions of social agents (e.g., enterprises), the objectives they pursue, the roles they play, the social relations they establish (commitments and corresponding claims), etc. The choice of using UFO for building UFO-S 
can be justified by successful application of UFO in previous works to evaluate, redesign, and ground ontologies, languages, and frameworks of several research areas, such as Software Engineering, Conceptual Modelling, and Enterprise Modelling (e.g., [1][2][16][36]). Further, UFO-S itself has been used to analyse the structuring principles of serviceoriented architectures [33] and the ArchiMate language concepts at the business layer [32].

In order to represent UFO-S, a UML profile that incorporates the foundational distinctions in UFO was used (OntoUML [15]). Table I presents the OntoUML stereotypes used in this paper. (Note that the term "universal" in UFO denotes types in conceptual modelling.)

TABLE I. A SUBSET OF ONTOUML STEREOTYPES USED IN UFO-S

\begin{tabular}{|c|c|}
\hline Stereotype & Corresponding Concept in UFO \\
\hline$<<$ category $>>$ & Category \\
\hline$<<$ collective $>>$ & Collective Universal \\
\hline$<<$ rolemixin $>>$ & Role Mixin \\
\hline$<<$ mode $>>$ & Mode Universal \\
\hline$<<$ relator $>>$ & Relator Universal \\
\hline$<<$ event $>>$ & Event Universal \\
\hline
\end{tabular}

The stereotypes in OntoUML correspond to ontological distinctions put forth by the (UFO). For instance, a class stereotyped as a category represents a rigid concept, i.e., a class that applies necessarily to its instances (throughout their entire existence). A role (mixin), in turn, is an anti-rigid concept, applying contingently to its instances (e.g., a Person can cease to be a Student and still exist). A role is also relational dependent, i.e., it defines contingent properties exhibited by an entity in the scope of a relationship. A relator class represents a reified relationship, and aggregates the properties (modes) that characterize entities in that relationship. Events are occurrences in a broad sense (encompassing complex events, atomic events, actions, interactions). (For further information on the distinctions underlying OntoUML, see [15]).

The use of the well-founded OntoUML profile for modelling UFO-S leverages the conceptual distinctions in UFO as well as the tool support already developed for OntoUML; This includes formal verification of the model (guaranteeing that the models put forth here are compliant with the UFO ontological constraints), validation of the model via visual simulation (relying on an OntoUML infrastructure developed on top of the Alloy Analyzer [3]) as well as the systematic implementation of UFO-S in computational level languages such as OWL [40]. In order to ensure precision of interpretation, we add axioms in firstorder logic to the UFO-S OntoUML models as a means to reflect the relevant constraints that are not directly implied by the OntoUML models (these are omitted here; the reader can refer to [31] for further details on the axiomatization of UFO-S).

UFO-S was designed aiming at a broad account for services independently of a particular application domain. It was also intended to harmonize different notions of service present in the literature, encompassing the notion of social commitments, which is deemed essential in UFO-S to account for complex service phenomena.
UFO-S focuses on the three basic phases of the service life-cycle [30]: (i) service offer (when a service is presented and made available to a target customer community), (ii) service negotiation (when providers and customers negotiate in order to establish an agreement), and (iii) service delivery (when actions are performed to fulfil a service agreement).

Figure 3 presents a UFO-S model fragment regarding service offer. A service offer is an event (e.g., the registration of a service provider organization in a chamber of commerce) that results in the establishment of a service offering, which mediates the social relations between the service provider and the target customer community. A service offering is composed of service offering commitments from the service provider towards the target customer community, and the corresponding service offering claims from the target community towards the service provider. Service offering commitments are metacommitments (i.e., they are commitments to accept commitments), since they refer to commitments that can be established later in the negotiation phase. The content of the service offering commitments and claims may be described in service offering descriptions (e.g., folders, registration documents in a chamber of commerce, and artefacts in software service registries).

Service provider is the role played by agents (e.g., physical agents such as persons, and social agents such as organizations [16]) when these agents commit themselves to a target customer community by a set of offering commitments. Target customer community is a collective that refers to the group of agents that constitute the community to which the service is being offered. Target customer is the role played by agents when they become members of the target customer community, and, as a consequence, have claims for the fulfilment of the commitments established by the agent playing the role of service provider.

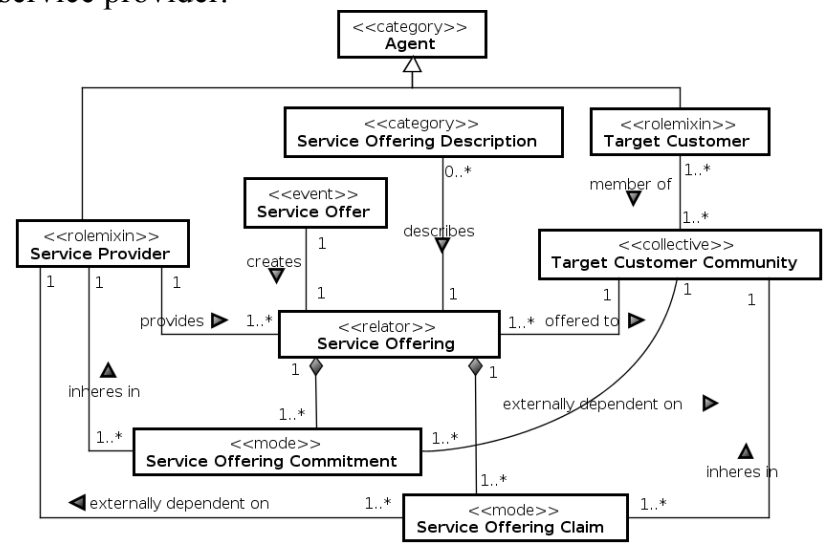

Figure 3. UFO-S service offer.

Once a service is offered, service negotiation may occur. Figure 4 presents UFO-S model fragment of this phase. Service negotiation is an event involving a target customer and a service provider. If service negotiation succeeds, a service agreement is established, and the service provider starts to play the role of hired service provider, while the target customer starts to play the role of service customer. 
A service agreement mediates the social relations between service customer and hired service provider, being composed of commitments and claims. Service agreements involve not only commitments from the hired service provider towards the service customer, but may also involve commitments from the service customer towards the hired service provider (e.g., the commitment to pay). Hired provider commitments and claims are properties that inhere in a hired service provider and are externally dependent on a service customer. Service customer commitments and claims are properties that inhere in a service customer and are externally dependent on a hired service provider. The content of commitments/claims of a service agreement may be described in a service agreement description (e.g., contract).

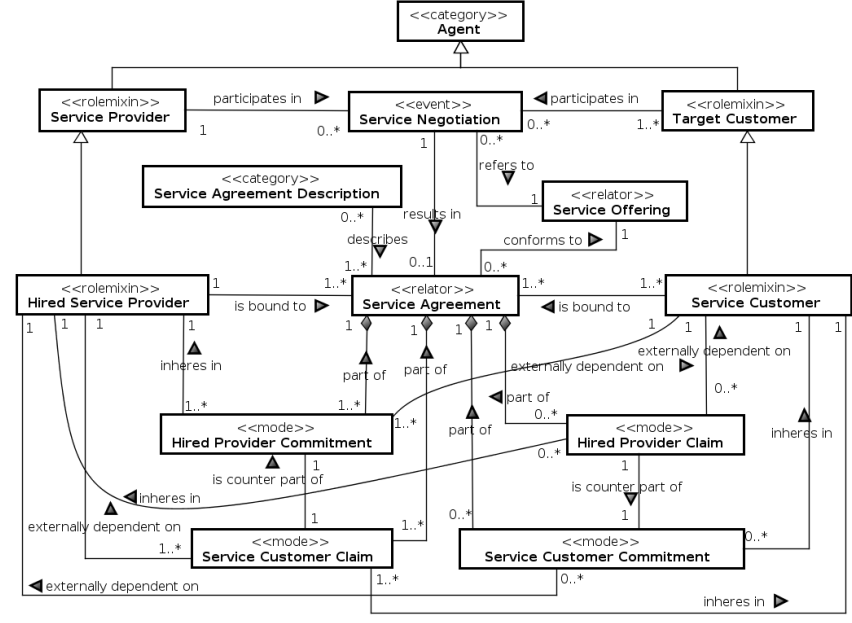

Figure 4. UFO-S service negotiation.

When a service agreement is established, the service customer delegates a goal/plan achievement/execution to the hired service provider. Thus, the mutual service commitments/claims established in the service agreement will drive service delivery. Figure 5 shows the fragment of UFO-S concerning service delivery. It makes explicit how provider and customer actions/interactions that are part of service delivery are motivated by commitments they have established as part of service agreement.

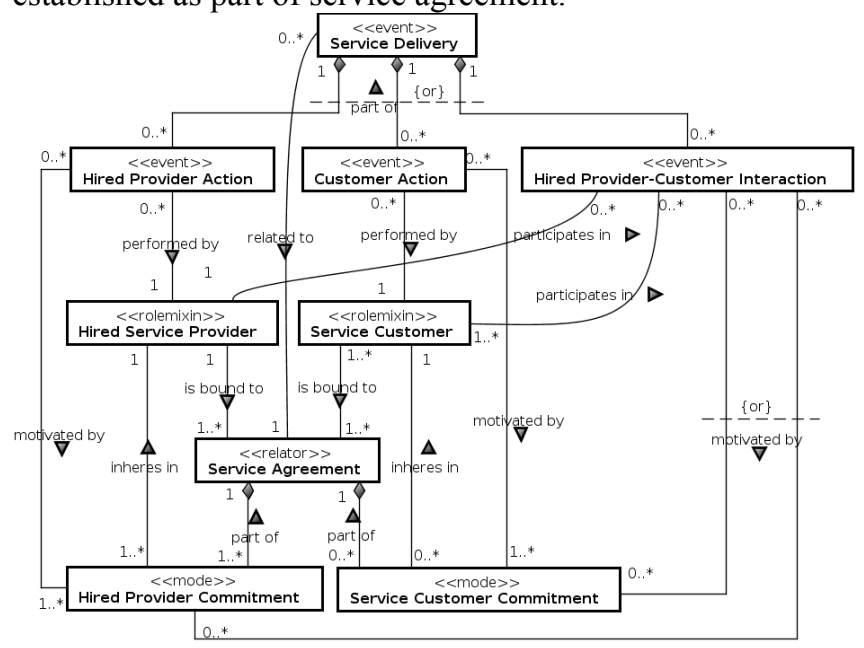

Figure 5. UFO-S service delivery

\section{AlignMent with UFO-S}

As highlighted in section II, there is increasing need to develop a precise expression of eHealth service semantics as part of a broader computable semantic interoperability approaches in eHealth. Such an expression is needed for those involved in the design and building of eHealth systems including those concerned with reasoning about eHealth services to better support reuse and service validation and safety at design time as well as service discovery at run time. This section analyses expressiveness of the SHO in terms of the UFO-S concepts, in order to ensure a sound ontological foundation to the SHO.

Previous two sections suggest that there is a great deal of similarity in coverage and intent between SHO and UFO-S, although they are developed by two different communities, namely, the eHealth standard developers and ontology/conceptual-modelling experts. However, there are also some difference is in the underpinning formalism. UFO$\mathrm{S}$ employs a stronger formalism owing to the use of the OntoUML profile with the accompanying formal semantics, as mentioned in section III. This formalism allows for the UFO-S models to be interpreted unambiguously - both by means of a formalization for the underlying UFO in modal logic presented in [15] as well as by means of an automated transformation of OntoUML diagrams into Alloy [3]. Further, UFO-S is grounded in a foundational ontology (UFO) while SHO is based on several SOA standards and RM-ODP foundations. These are specified in normative text and accompanied by the use of UML notation to support description of the modelling concepts and their relationships. Finally, as opposed to UFO-S, no axioms are provided in the SHO to further constrain possible interpretations of servicerelated concepts.

These similarities and differences motivate our interest into establishing the alignment of SHO and UFO-S in order to provide a stronger semantic foundation to $\mathrm{SHO}$, but also to test the coverage of UFO-S concerning eHealth service concepts. This section provides a further comparative analysis of these two ontologies.

Our comparative analysis led us to several key observations as described next. (Note that SHO concepts are identified in italics and UFO-S concepts are underlined.)

First, in the service offer phase, the differences in the approaches stem from the choice of concepts to capture commitments that will drive the rest of the service lifecycle. In this phase, UFO-S explicitly considers that a service provider performs a service offering action that results in a service offering from the service provider towards the target service customers (which may be described in a service offering description). On the other hand, SHO relies primarily on the notion of Business Service Description for the service offer stage. A first observation is that the definition of Business Service Description in SHO allows us to interpret it according to UFO-S as: (i) a description of a type of service offering, or, (ii) a description of a particular service offering. If it describes a type of service offering (say "dental services" in general), then no commitments exist, i.e., no particular provider is committed to offer the service 
that is described. If it is intended to describe a particular service offering (say "dental services that are provided by Dr. John Smith"), then, still, the sole existence of the description does not entail a commitment of a provider to comply with the description. This is because the descriptions may not have been advertised, published or registered for discovery, i.e., there have been no actions by the provider that could count as a service offer. This suggests that the SHO can be extended to explicitly account for these actions. This is possible by explicitly including the notion of commitment from RM-ODP in SHO, which results in creating an obligation for a party, in this case the service provider. Further, the apparent ambiguity in the interpretation of business service description may be addressed by stipulating a particular interpretation. The interpretation that seems to favour the expressiveness of $\mathrm{SHO}$ is to understand a business service description as a description of a type of service offering (interpretation (i) above). In this case, the commitment action entails the instantiation of the type of service offering described, determining the provider that is committed (e.g., through this action it is possible for "Dr. John Smith" becomes committed to offer a "dental service"). In this solution a service offering description would consist of a Business Service Description with additional information to determine the provider that is committed. The notion of type of service offering also helps us to explain what is meant by a services taxonomy in SHO: this would actually be a taxonomy of service offering types, starting with high level categories and branching into more purpose-specific categories.

Second, the SHO approach does not distinguish between different policies that may apply to the agents in different phases of service lifecycle. For example, in SHO, there is no distinction between what UFO-S refers to as service provider and hired service provider roles and between target customer and service customer. Each of these roles would imply different commitment and claim policies. It may be useful to provide this distinction in next release of $\mathrm{SHO}$, but this would need to be motivated by several use cases, which is a typical HL7 standardisation practice.

Third, the current release of SHO was developed with an aim of providing a rich set of policy constraints that characterise eHealth requirements, through the identification of key deontic policies in the model, i.e. obligations, permissions and prohibitions. The deontic policies underpin healthcare policies such as privacy, consent, duty of care and accountability, and will be informed by related work in HL7 [22]. This highlighted the need for the UFO-S to its current commitment-claim paradigm with a broader set of deontic policies. (This is probably to be done through an extension of the social layer of UFO, as deontic aspects are relevant to social reality in general and not only to services.) The ability to account for deontic aspects beyond commitments and claims would allow UFO to explain what happens when commitments are not honoured (which is currently outside the scope of the model and only discussed in text). In SHO, this is possible using the primitive notions of obligation and permission (from RM-ODP) as well as the so-called contrary-to-duty obligations - which can be regarded as a permission for one party to imply an obligation on another party in the presence of violations [8] (e.g. permission of a Target Customer to raise obligation of a Service Provider).

Fourth, the SHO specification, unlike UFO-S, includes a computational view on service to support IT experts in expressing computational service concept in their solution architecture, irrespectively of specific technology choices such as SOAP or REST (We have omitted the computational service definitions and models from Section II, and have focused on business services instead.) Although UFO-S does not provide explicit concept for a computational (technical) service, such a concept can be expressed using UFO-S, whereby a service offering description is often reduced to descriptions of operations or sets of operations that are typically characterized by a pair of interaction types and constraints on them (possibly relating several operations) [30]. An important difference between UFO-S and SHO with respect to the account of computational services is that for UFO-S service phenomena involve necessarily commitments between intentional agents (which are capable of establishing commitments). This means that while in SHO computational objects are considered providers and consumers ("requestor" and "offerer") of services, in UFO-S, these would be the agents responsible to guarantee the operation of such computational objects to honour commitments. These agents may employ other resources (such as, e.g., the labour of systems operators) in order to honour such commitments.

Fifth, the analysis has also identified several areas where the SHO conceptual model needs to be updated, including the development of precise constraints related to how party or active enterprise objects can fulfil provider and customer roles, as well as update of a number of cardinality constraints in the model.

Finally, it would be of value to express the SHO conceptual model using OntoUML semantics to check validity of our comparative analysis and we are planning to work on this in next stage of this research.

Table II summarizes the alignment. The table is structured in terms of the phases of service lifecycle of UFO$\mathrm{S}$ (service offer, service negotiation and service delivery).

TABLE II. SUMMARY OF UFO-S AND SHO ALIGNMENT

\begin{tabular}{|l|l|}
\hline \multicolumn{1}{|c|}{ UFO-S Concept } & \multicolumn{1}{|c|}{ SOA Healthcare Concepts } \\
\hline$\underline{\text { Agent }}$ & Service Offer phase in UFO-S \\
\hline Service Provider & Provider \\
\hline$\underline{\text { Target Customer }}$ & $\begin{array}{l}\text { No explicit concept - may be mentioned in Business } \\
\text { Service Descriptions }\end{array}$ \\
\hline$\underline{\text { Target Customer }}$ & $\begin{array}{l}\text { No explicit concept - may be mentioned in Business } \\
\text { Service Descriptions }\end{array}$ \\
\hline Community & $\begin{array}{l}\text { No explicit concept - but a special kind of business } \\
\text { event can be added and linked to Business Service } \\
\text { Description (e.g., a publication and/or advertisement } \\
\text { of a business service description) }\end{array}$ \\
\hline Service Offering & $\begin{array}{l}\text { No explicit concept - although a Business Service } \\
\text { Description representing a type of service offering } \\
\text { may be instantiated, and the result of this could be } \\
\text { captured in a new description }\end{array}$ \\
\hline Service Offering & $\begin{array}{l}\text { No explicit concept }- \text { but can be interpreted as the } \\
\text { existence of a published Business Service Description }\end{array}$ \\
\hline
\end{tabular}




\begin{tabular}{|c|c|}
\hline & with information of a specific provider identifier \\
\hline $\begin{array}{l}\text { Service Offering } \\
\text { Commitment }\end{array}$ & $\begin{array}{l}\text { Obligation of a Provider (to potential Consumers); } \\
\text { this is done indirectly via constraint of Provider's } \\
\text { behaviour; note that the SHO can adopt the normative } \\
\text { definition of commitment from RM-ODP, defined as } \\
\text { 'an action resulting in an obligation by one or more of } \\
\text { the participants in the act to comply with a rule or } \\
\text { perform a contract.' [25] }\end{array}$ \\
\hline ffering & $\begin{array}{l}\text { Implicit in the obligation of a provider, in which a } \\
\text { Consumer is mentioned as intended beneficiary. }\end{array}$ \\
\hline \multicolumn{2}{|r|}{ Service Negotiation phase in UFO-S } \\
\hline Servic & $\begin{array}{l}\text { Business Service Contract. Business service contract } \\
\text { implies a relationship between service provider and } \\
\text { service consumer and in that case is a type of UFO } \\
\text { relator, like UFO-S service agreement. }\end{array}$ \\
\hline$\frac{\text { Servi }}{\text { Desci }}$ & $\begin{array}{l}\text { No explicit concept - can be added as a special kind } \\
\text { of information artefact }\end{array}$ \\
\hline $\begin{array}{l}\text { Hired Service } \\
\text { Provider }\end{array}$ & $\begin{array}{l}\text { Does not exist but it would be useful to distinguish } \\
\text { from Service Provider }\end{array}$ \\
\hline Customer & Const \\
\hline$\frac{\text { Hired provider }}{\text { commitment }}$ & $\begin{array}{l}\text { Does not exist but may be useful to distinguish from } \\
\text { Service Offering commitments; can be modelled } \\
\text { using deontic logic concepts of obligations }\end{array}$ \\
\hline er claim & $\begin{array}{l}\text { Does not exist but may be useful to distinguish from } \\
\text { Service provider claims. }\end{array}$ \\
\hline $\begin{array}{l}\text { Service Customer } \\
\text { claim }\end{array}$ & Does not exist but may be useful to explicitly model \\
\hline $\begin{array}{l}\text { Service Customer } \\
\text { commitment }\end{array}$ & Does not exist but may be useful to explicitly model \\
\hline \multicolumn{2}{|r|}{ Service Delivery phase in UFO-S } \\
\hline Servi & Serv \\
\hline r Action & by Consumer \\
\hline Hired Provider & Part of Service performed by Provider \\
\hline $\begin{array}{l}\text { Hired Provider - } \\
\text { Customer interac }\end{array}$ & $\begin{array}{l}\text { Part of Service performed by Con } \\
\text { interaction }\end{array}$ \\
\hline
\end{tabular}

\section{DISCUSSION}

The term "ontology" has been used in computer science to denote artefacts designed with different purposes, leading to a variety of characteristics, including different kinds of representation approaches (modelling languages, formalisms, etc.), different scopes, levels of generality and abstraction.

We believe that these artefacts may have different roles to play in standardization efforts, owing to their various characteristics. We can group these different approaches to "ontology" in two broad categories according to their purpose [13]:

- reference ontologies, whose purpose is to facilitate understanding and human communication (such as $\mathrm{SHO}$, UFO-S, and the Open Group SOA Ontology [38]); and, - computational ontologies (also called lightweight or operational ontologies [6][13]), whose purpose is to facilitate automated inference and run-time support.

Reference ontologies postulate the types of entities that are considered to exist in a certain universe of discourse and can be understood as a descriptive theory of that universe of discourse (e.g., service phenomena, genealogy, social reality, law, biology). Reference ontologies should preferably be specified using highly expressive languages and formalisms (such as logics or logic-based formalisms such as SBVR [35]) in order to ensure precision of interpretation, and thereby increase the change of success in communication and shared understanding. They should be specified in such a way as to guide the interpretation of the concepts to ensure the phenomenon they represent is precisely captured, ruling out inadmissible interpretations, thereby reducing the possibility of false agreement [11] (which occurs when we use the same terms with different meaning and do not notice). UFO-S was built on this premise, and therefore is specified in a language (OntoUML) that includes important distinctions between types that apply necessarily to its instances (categories, kinds, subkinds, among others) and those that apply contingently (roles, phases, among others). Further, the language semantics is also formalized to allow for unambiguous interpretation of models.

Computational ontologies, in contrast, are mainly targeted to build knowledge bases in computational applications. They are represented in languages that sacrifice expressiveness for the sake of computational tractability (e.g., RDF(s) and OWL variants), affecting their ability to rule out inadmissible interpretations. For example, OWL cannot express the modal properties of rigidity, anti-rigidity, external dependence and others underlying UFO. Since the main purpose of computational ontologies is to serve as a schema for knowledge bases (even distributed ones in the case of the Semantic Web), they do not include concepts that, albeit relevant for shared understanding, would not be instantiated in a knowledge base. An example of such a concept in SHO is behaviour. Behaviour is key to the conceptualization of SHO, however, it would only be included in a Semantic Web ontology if one was interested in tracking behaviour after its occurrence (as "records" of behaviour could be in a knowledge base). Examples of semantic web ontologies concerning services are OWL-S [28] and WSMO [5]. They are both focused on the description of technical services to serve in automated service discovery and composition tasks.

We believe that a principled approach to standardization efforts should be based on the definition of expressive reference ontologies to capture key conceptual distinctions needed for the standardization effort. Computational ontologies could then be generated from reference ontologies in order to support ontology-aware implementations and interoperability formats (see, e.g., the approach in [40]). An immediate benefit of this approach is that the computational ontologies are structured following the principles that guide the engineering of reference ontologies (such as clarity and precision). We believe that the benefits of employing reference ontologies for the development of well-founded standards should ultimately be extended to conforming system implementations, as they should have better conceptual integrity - a key factor for system success [4].

Ontologies can also be further classified according to their level of generality, continuously range from foundational (or top-level) ontologies, through core ontologies, domain ontologies and application ontologies [7]. Foundational ontologies describe very general concepts like space, time, object, event, etc., and are independent of particular domains or problems [10]. Core and domain ontologies describe concepts related to a (more or less) generic domain, sometimes specializing concepts of a toplevel ontology [37]. Application ontologies, are placed at the 
other end of the spectrum with respect to foundational ontologies, describing concepts related to a particular application [10]. Since these categories of ontologies form a continuum, the borderline between them is not clearly defined [7]. Given the generality level required of standardization efforts, we expect that most ontologies produced in such efforts to be core and domain ontologies. We argue that ontologies in standards should be aligned with foundational ontologies (such as UFO [15], DOLCE [29], BFO [9], IDEAS upper ontology [23]) in order to define pervasive concepts that are specialized in specific domains of interest. This should ensure that they reuse stable highquality foundations. An example of successful standardization effort that follows this strategy is DoDAF [39], whose conceptual model is based on the IDEAS upper ontology. The availability of foundations for standards also facilitates the comparison and alignment of standards (or families of standards). This was the case in the comparison of SHO and UFO-S reported here; the work was facilitated because SHO inherits explicit definitions of basic foundational concepts from RM-ODP Part 2 [24] (such as object, behaviour, policy, roles, party, commitment, etc.). These definitions have allowed us find comparable UFO concepts (such as, e.g., UFO agents corresponding to the ODP notion of party).

\section{FINAL CONSIDERATIONS}

The purpose of the HL7 SOA Healthcare Ontology (SHO) is to serve as a shared reference conceptual model, which can guide the definition of service-related aspects of future eHealth standards and solutions. One example is a need to better position new HL7 standard called Fast Health Interoperability Resources (FHIR) in the context of behaviour semantics, particularly in terms of eHealth services and processes.. The main focus of FHIR is on the specification of eHealth resources using XML or JSON while making use of REST infrastructure to access and exchange such resources. Recent discussions on HL7 lists have indeed identified the need to express more complex eHealth interactions, including orchestration between participants, using FHIR concepts. We anticipate that such developments will require a well-articulated approach to specifying services, and we believe that our proposal provides early steps in this direction.

The HL7 SOA Healthcare Ontology intent is to establish a shared language for those who will build and operate systems for eHealth services. In this respect, this ontology has a similar intent as UFO-S, although driven from eHealth requirements. In this paper we have tested the $\mathrm{SHO}$ against UFO-S as a reference ontology and provided few suggestions to improve it in order to be better grounded on a solid conceptual foundation.

Our approach has been to analyse the SHO service concepts in terms of the UFO-S ontology. This has served two purposes: (i) to ensure that the HL7 SOA Healthcare Ontology is grounded on a core reference ontology that approaches services from a broad perspective, addressing behavioural aspects of services including commitment aspects; and, (ii) to establish requirements for further development of UFO-S and HL7 SOA Healthcare Ontology.

The analysis has revealed that certain notions of commitments/claims in UFO-S can guide the further refinement of $\mathrm{HL} 7 \mathrm{SHO}$, introducing notions of hired service provider commitments and claims as well as service customer commitments and claims. The analysis has also revealed that the definition of business service description deserves some further attention in $\mathrm{SHO}$, as the current text makes it ambiguous (denoting either a type of service offering or a particular service offering). Further, while HL7 SHO's view on services does not reify service offering (commitments/claims) in its current version, such commitments and claims can be addressed using policy concepts (also a heritage of RM-ODP).

With respect to further requirements for UFO-S, the main point of attention is the full incorporation of deontic aspects behind service commitments. While service commitments/claims have an inherent deontic perspective, there is currently no explicit account for obligations, prohibitions, permissions and other deontic notions. Having an explicit account for such notions would allow us to capture the semantics of service commitments in a more comprehensive way.

Further developments that apply to both UFO-S and HL7 SHO include the need to expand on the descriptions of service offerings (business service descriptions for HL7 $\mathrm{SHO}$ ) and service agreements (service contract descriptions of SHO). This should serve as a basis for data formats that can be used by providers to advertise service offerings (which will in turn guide service discovery and composition) and also as a basis for service agreement monitoring. We believe these approaches can be anchored on existing contract languages and monitoring approaches.

Finally, we should remark that service science and service-oriented enterprise architecture can only mature as disciplines if solid conceptual foundations are available. Harmonizing and positioning the various (competing and complementary) perspectives as well as the standards, specifications and frameworks that approach services from these various perspectives is an important contribution to the discipline. This task is only feasible if the various standards, specifications and frameworks make explicit their underlying conceptualizations.

\section{ACKNOWLEDGEMENTS}

This research is partially funded by the Brazilian Research Funding Agencies FAPES (grant number 59971509/12), CNPq (grants number 310634/2011-3 and 485368/2013-7) and CAPES.

\section{REFERENCES}

[1] J. P. A. Almeida and G. Guizzardi, "An Ontological Analysis of the Notion of Community in the RM-ODP Enterprise Language," Computer Standards \& Interfaces, vol. 34, pp. 257-268, 2012.

[2] C. Azevedo, J. P. A. Almeida, M. J. Van Sinderen, D. Quartel, and G. Guizzardi, "An Ontology-Based Semantics for the Motivation Extension to ArchiMate," in 15th IEEE International Conference on Enterprise Computing (EDOC), 2011, Helsinki, Finland, pp. 25-34. 
[3] A. B. Benevides, G. Guizzardi, B. F. B. Braga, and J. P. A. . Almeida, "Validating Modal Aspects of OntoUML Conceptual Models Using Automatically Generated Visual World Structures," Journal of Universal Computer Science (Print), vol. 16, pp. 2904-2933, 2011.

[4] F. Brooks, The Mythical Man-Month, Addison-Wesley, 1975.

[5] J. de Bruijn, C. Bussler, J. Domingue, D. Fensel, M. Hepp, M. Kifer, B. König-Ries, J. Kopecky, R. Lara, E. Oren, A. Polleres, J. SciCluna, and M. Stollberg, "Web Service Modeling Ontology (WSMO). Final Draft.," 2006. [Online]. Available: http://www.wsmo.org/TR/d2/v1.3/.

[6] R.A. Falbo, G. Guizzardi, A. Gangemi, and V. Presutti, "Ontology Patterns: Clarifying Concepts and Terminology," in 4th Workshop on Ontology Patterns (WOP2013), 12th International Semantic Web Conference (ISWC 2013), Sydney, Australia, 2013.

[7] R.A. Falbo, M.P. Barcellos, J.C. Nardi, and G. Guizzardi, "Organizing Ontology Design Patterns as Ontology Pattern Languages," in 10th Extended Semantic Web Conference (ESWC), 2013.

[8] G. Governatori and Z. Milosevic, "A formal analysis of a business contract language", International Journal of Cooperative Information Systems (IJCIS), special issue on Enterprise Distributed Computing, vol. 15, no. 4, December 2006.

[9] Grenon, P., Smith, B., and Goldberg, L. "Biodynamic ontology: applying BFO in the biomedical domain." Studies in health technology and informatics, 102(ii), pp. 20-38, 2004.

[10] N. Guarino, "Formal Ontology and Information Systems". In Proceedings of FOIS'98, IOS Press, 1998, pp. 3-15..

[11] N. Guarino, 'The Ontological Level', in R. Casati, B. Smith and G. White (eds.), Philosophy and the Cognitive Science. Holder-PivhlerTempsky, Vienna: pp. 443-456, 1994.

[12] R. S. S. Guizzardi, "Agent-Oriented Constructivist Knowledge Management," University of Twente, 2006.

[13] G. Guizzardi, "On ontology, ontologies, conceptualizations, modeling languages, and (meta) models", in Vasilecas, O., Eder, J.,Caplinskas, A. (Eds.), Databases and Information Systems IV, IOS Press, Amsterdam, 2007, pp. 18-39.

[14] G. Guizzardi, "On Ontology, ontologies, Conceptualizations, Modeling Languages, and (Meta)Models," in Frontiers in Artificial Intelligence and Applications, Databases and Information Systems IV, vol. 15, O. Vasilecas, J. Edler, and A. Caplinskas,. Amsterdam, IOS Press, 2007, pp. 18-39.

[15] G. Guizzardi, "Ontological Foundations for Structural Conceptual Models," University of Twente, 2005.

[16] G. Guizzardi, R. A. Falbo, and R. S. S. Guizzardi, "Grounding software domain ontologies in the Unified Foundational Ontology (UFO): the case of the ODE software process ontology," in Proceedings of the XI Iberoamerican Workshop on Requirements Engineering and Software Environments, 2008, pp. 244-251.

[17] G. Guizzardi, G. Wagner, R. de A. Falbo, R. S. S. Guizzardi, and J. P. A. Almeida, "Towards Ontological Foundations for the Conceptual Modeling of Events," in 32th International Conference on Conceptual Modeling (ER), 2013, pp. 327-341.

[18] HealthIT.gov, Health Information Exchange (HIE), Standards \& Interoperability, Available https://www.nehta.gov.au/implementation-resources/ehealthfoundations/EP-1144-2007

[19] Health Level Seven International, "HL7 Service-Aware Interoperability Framework: Canonical Definition, Release 1, HL7 Service-Aware Interoperability Framework: Canonical Definition Specification, R2, SAIF", Available at www.hl7.org/implement/standards/product brief.cfm?product id=3

[20] Health Level Seven International, http://www.hl7.org
[21] Health Level Seven International, "Healthcare SOA Ontology, Release 1, May 2013, HL7 For Comment Only Ballot, Sponsored by: SOA WG Healthcare Services Specification Program", Available at http://hssp-infrastructure.wikispaces.com/HSSP+SOA+Service+ Ontology

[22] Health Level Seven International (HL7-SOA and HL7-Security Technical Committees), "PASS Architecture Overview", Available at http://hssp-security.wikispaces.com/PASS+Architecture+Overview

[23] IDEAS Group, Available at http://www.ideasgroup.org

[24] ITU-T/ISO, “ITU-T X.902 | ISO/IEC 10746-2, Information Technology Open Distributed Processing Reference Model Foundations", 2010.

[25] ITU-T/ISO, "ITU T Rec. X.911 | ISO/IEC 15414: Enterprise language DIS for ITU T Recommendation X.911| ISO/IEC 15414 Amd 1", 2013.

[26] P.F. Linington, Z. Milosevic, A. Tanaka and A. Vallecillo, Building Enterprise Systems with ODP, An Introduction to Open Distributed Processing, Chapman \& Hall/CRC Press, 2011.

[27] National E-Health Transition Authority (NEHTA), "The NEHTA Interoperability Framework (IF)", Available at http://www.healthit.gov/providers-professionals/standardsinteroperability

[28] D. Martin, M. Burstein, J. H. O. Lassila, D. McDermott, and E. Al., "OWL-S: Semantic markup for web services", 2004. Available at: http://www.w3.org/Submission/OWL-S/

[29] C. Masolo, S. Borgo, A. Gangemi, N. Guarino, A. Oltramari., "WonderWeb DeliverableD18. Ontology Library," Padova-Italy, 2003.

[30] J. C. Nardi, R. Falbo, J. P. A. Almeida, G. Guizzardi, L. F. Pires, M. van Sinderen, and N. Guarino, "Towards a Commitment-based Reference Ontology for Services," in 17th IEEE International EDOC Conference (EDOC), IEEE CS Press, 2013.

[31] J. C. Nardi, C. M. Fonseca, J. P. A. Almeida, and R. Falbo, "UFO-S Formalization," Technical Report, 2014. Available at http://nemo.inf.ufes.br/files/ufo s techreport.pdf

[32] J. C. Nardi, R. Falbo and J.P.A. Almeida, "An Ontological Analysis of Service Modeling at ArchiMate's Business Layer", in 18th IEEE International EDOC Conference (EDOC), IEEE CS Press, 2014.

[33] J. C. Nardi, R. Falbo and J.P.A. Almeida, "Revealing Service Commitments in Service-Oriented Enterprise Architecture", in 6th Workshop on Service-Oriented Enterprise Architecture for Enterprise Engineering (SoEA4EE 2014), 18th IEEE International EDOC Conference Workshops (EDOCW), 2014.

[34] OMG, "Model Driven Architecture", http://www.omg.org/mda/

[35] OMG, "Semantics of Business Vocabularies and Rules", Available at http://www.omg.org/spec/SBVR/

[36] P. S. Santos Jr., J. P. A. Almeida, and G. Guizzardi, "An ontologybased analysis and semantics for organizational structure modeling in the ARIS method," Information Systems (Oxford), pp. 690-708, 2012.

[37] A. Scherp, C. Saathoff, T. Franz, and S. Staab, "Designing core ontologies," Applied Ontology, vol. 6, no. 3, pp. 177-221, 2011.

[38] The Open Group, "Service-Oriented Architecture Ontology", The Open Group, 2009.

[39] U.S. Department of Defense, "DoD Architecture Framework Version 2.02", 2010, Available at http://dodcio.defense.gov/dodaf20.aspx

[40] V. Zamborlini and G. Guizzardi, "On the Representation of Temporally Changing Information in OWL," in Enterprise Distributed Object Computing Conference Workshops (EDOCW) VORTE, 2010, pp.283-292. 